\title{
Forced oscillation measurements of seismic wave attenuation and stiffness moduli dispersion in glycerine-saturated Berea sandstone
}

\author{
Samuel Chapman ${ }^{1,2 *}$, Jan V. M. Borgomano ${ }^{2}$, Hanjun Yin ${ }^{2,3}$, Jerome Fortin ${ }^{2}$ \\ and Beatriz Quintal ${ }^{1}$ \\ ${ }^{1}$ Institute of Earth Sciences, University of Lausanne, 1015 Lausanne, Switzerland, ${ }^{2}$ Laboratoire de Géologie, Ecole Normale \\ Supérieure/CNRS UMR 8538, PSL Research University, 75005 Paris, France, and ${ }^{3}$ State Key Laboratory of Petroleum Resources and \\ Prospecting, China University of Petroleum-Beijing, 102249 Beijing, China
}

Received October 2017, revision accepted October 2018

\begin{abstract}
Fluid pressure diffusion occurring on the microscopic scale is believed to be a significant source of intrinsic attenuation of mechanical waves propagating through fully saturated porous rocks. The so-called squirt flow arises from compressibility heterogeneities in the microstructure of the rocks. To study squirt flow experimentally at seismic frequencies the forced oscillation method is the most adequate, but such studies are still scarce. Here we present the results of forced hydrostatic and axial oscillation experiments on dry and glycerine-saturated Berea sandstone, from which we determine the dynamic stiffness moduli and attenuation at micro-seismic and seismic frequencies $(0.004-30 \mathrm{~Hz})$. We observe frequency-dependent attenuation and the associated moduli dispersion in response to the drained-undrained transition $(\sim 0.1 \mathrm{~Hz})$ and squirt flow $(>3 \mathrm{~Hz})$, which are in fairly good agreement with the results of the corresponding analytical solutions. The comparison with very similar experiments performed also on Berea sandstone in addition shows that squirt flow can potentially be a source of wave attenuation across a wide range of frequencies because of its sensitivity to small variations in the rock microstructure, especially in the aspect ratio of micro-cracks or grain contacts.
\end{abstract}

Key words: Attenuation, Rock physics.

\section{INTRODUCTION}

Porous rocks saturated with fluids can strongly attenuate seismic waves. Different forms of fluid pressure diffusion (FPD), which is also commonly referred to as wave-induced fluid flow, are thought to be the primary intrinsic mechanism for seismic wave attenuation (e.g. Pride, Berryman and Harris 2004). FPD arises predominantly from contrasts in compressibility either in the solid matrix of the rock, for instance between compliant grain contacts and stiff pores, or in the saturating fluids, such as a heterogeneous distribution of water and gas. In response to such compressibility

\footnotetext{
*E-mail: samuel.chapman@unil.ch
}

contrasts, seismic waves induce pressure gradients, resulting in FPD associated with displacement of the viscous fluid and the conversion of the mechanical energy of the wave into heat due to viscous friction. The frequency dependence of the associated seismic attenuation depends strongly on the spatial distribution and scale of the heterogeneities in the rock matrix, as well as on the saturating fluids (Masson and Pride 2007, 2011; Müller, Toms-Stewart and Wenzlau 2008). A direct consequence of the frequency-dependent attenuation is that the corresponding stiffness modulus of the rock will also be frequency-dependent.

Much focus has been given to squirt flow, FPD arising from microscopic compressibility heterogeneities in the rock, as one of the dominant mechanisms for wave 
attenuation in fluid-saturated rocks. Numerous theoretical models (e.g. O'Connell and Budiansky 1977; Mavko and Jizba 1991; Chapman, Zatspin and Crampin 2002; Gurevich et al. 2010; Adelinet, Fortin and Gueguen 2011) have been developed to explain laboratory observations at sonic and ultrasonic frequencies. The characteristic frequency of squirt flow is inversely proportional to fluid viscosity (Jones 1986). Therefore, using high-viscosity fluids, such as glycerine, squirt flow has also been studied at seismic frequencies with the forced oscillation method (e.g. McKavanagh and Stacey 1974), allowing for a better resolution with respect to frequency of attenuation and modulus dispersion.

On a Fontainebleau sandstone sample fully saturated with glycerine, Pimienta, Fortin and Guéguen (2015a) observe an extensional mode attenuation peak at between 1 and $10 \mathrm{~Hz}$, which is reduced in amplitude with increasing effective stress. Subramaniyan et al. (2015) also measure the extensional mode attenuation and Young's modulus in Fontainebleau sandstone with similar properties, in this case varying the fluid viscosity by mixing water and glycerine. For the fully glycerine-saturated sample, they observe an attenuation peak in a similar frequency range and with comparable amplitude, supporting the observation of Pimienta et al. (2015a). In addition, Subramaniyan et al. (2015) use Gurevich et al.'s (2010) analytical solution for squirt flow to interpret their observations. However, the analytical solution consistently underestimates the attenuation magnitude measured in the laboratory. The broad attenuation peaks observed are attributed to a distribution of crack aspect ratios in the rock sample.

In a glycerine-saturated Berea sandstone sample, Mikhaltsevtich et al. (2015, 2016) measure the dynamic Young's modulus and Poisson ratio, from which they infer the bulk and shear moduli as well as the corresponding attenuation modes. By performing measurements at temperatures from $31^{\circ} \mathrm{C}$ to $23^{\circ} \mathrm{C}$, they observe a shift of the extensional mode attenuation peak from $\sim 2$ to $\sim 0.4 \mathrm{~Hz}$, associated with the reduction of the glycerine viscosity. Mikhaltsevtich et al. (2015) interpret the attenuation as being caused by squirt flow. Spencer and Shine (2016) also perform forced oscillation experiments on a fully saturated Berea sandstone sample, however only observing a partial attenuation curve. Similar to Mikhaltsevtich et al. (2016), the impact of fluid viscosity is studied, however instead of modifying the temperature, different fluids of varying degrees of viscosity are used. Pimienta et al. (2017) investigate frequency-dependent attenuation and modulus dispersion in four different types of sandstone, including Berea sandstone, under full water and glycerine saturation. In both Wilkenson and Bentheim sandstones, frequency-dependent attenuation is observed likely in response to squirt flow, showing a strong sensitivity to changes in effective pressure. However, in the Berea sandstone samples, frequency-dependent attenuation and dispersion in response to squirt flow is not verified in the considered frequency range.

Even though there has been a recent surge in the availability of laboratory data from fluid-saturated sandstones at seismic frequencies $(<100 \mathrm{~Hz})$, the overall understanding of the physical processes responsible for the frequency-dependent attenuation and modulus dispersion remains incomplete. Comparing experiments is especially challenging given the variation in microstructure between samples and the use of different saturating fluids. Furthermore, the analysis is considerably complicated by the impact or not of boundary conditions on the observed frequency-dependent attenuation, which is associated with the design of the experimental apparatus (e.g. Pimienta et al. 2016; Chapman and Quintal 2018).

To contribute to the available data and current knowledge and understanding in the field, we present in the following sections the results of forced hydrostatic and axial oscillation experiments on a Berea sandstone sample. The experiments were performed on the dry and fully glycerinesaturated sample for a range of effective stresses. We provide a description of the sample and the experimental conditions. The discussion of our results for the stiffness moduli dispersion and corresponding attenuation modes addresses the uncertainty in our measurements and focuses on the physical processes responsible for our observations, how the theoretical predictions compare to our observations and how our observations compare to those of Mikhaltsevtich et al. (2016) whose experiments are the most similar to ours.

\section{SAMPLES AND EXPERIMENTAL METHODOLOGY}

\subsection{Sample description}

Three samples with a $\sim 4 \mathrm{~cm}$ diameter and $\sim 8 \mathrm{~cm}$ length were cored from a block of Berea sandstone with poorly defined bedding planes, running subparallel to the samples vertical axis (Fig. 1). The Berea sandstone was purchased from Cleveland Quarries in the United States with a brine permeability estimated at $75-250 \mathrm{mD}$. We measured the glycerine permeability of sample BS-V5 at an effective stress of $2.5 \mathrm{MPa}$ by imposing a pressure gradient across the sample and measuring the associated flow. The porosity was determined with 


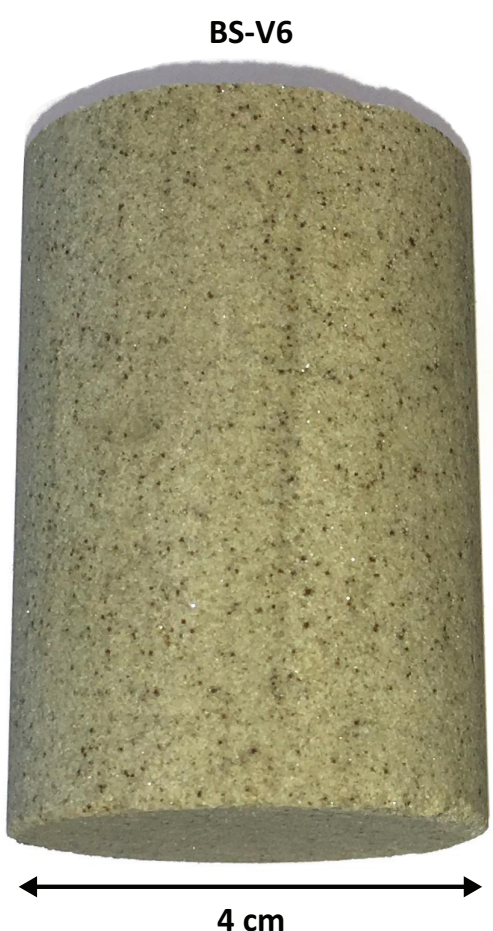

Figure 1 Photograph of sample BS-V6 with poorly defined bedding planes subparallel to the vertical axis. The data presented in this article are from experiments performed on sample BS-V5 cored from the same block as BS-V6.
Table 1 Sample properties

\begin{tabular}{ll}
\hline & BS-V4 to BS-V6 \\
\hline Glycerine permeability $(\mathrm{mD})$ & $58.7 \pm 1.1^{*}$ \\
Porosity $(\%)$ & 22.15 \\
Dry density $\left(\mathrm{kg} / \mathrm{m}^{3}\right)$ & $2087 \pm 7$ \\
\hline
\end{tabular}

"Measured at 2.5 MPa effective stress.

a pyknometer using a subsection of sample BS-V6. The dry density is the mean density determined from the dry masses and dimensions of the three samples. These properties are listed in Table 1. Kareem et al. (2017) performed an extensive characterization of Berea sandstone cores, also from Cleveland Quarries, showing that clays make up between 3\% and $9 \%$ of the bulk composition, with the predominant clay being kaolinite. The scanning electron microscopy and energy dispersion spectrometry (SEM-EDS) analyses on a portion of BS-V6 (Fig. 2) show that our sample is composed largely of quartz, with smaller amounts feldspar and clays, consistent with the analysis of Kareem et al. (2017).

\subsection{Experimental set-up - dynamic moduli and attenuation modes}

Forced oscillation measurements were performed in a tri-axial cell at ENS Paris. Borgomano et al. (2017) provide a detailed (a) SEM Image

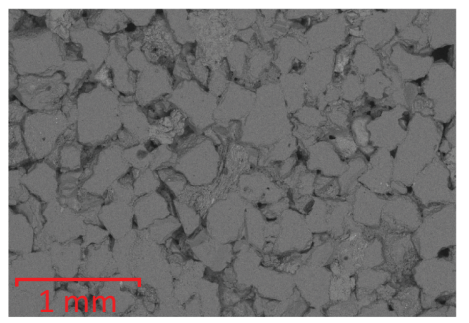

(d)

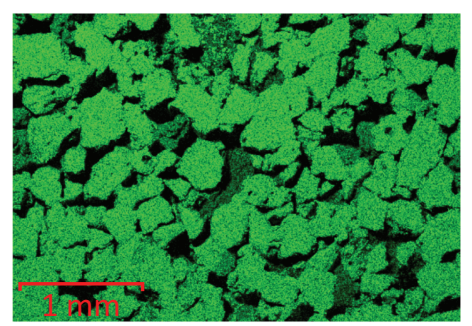

(b)

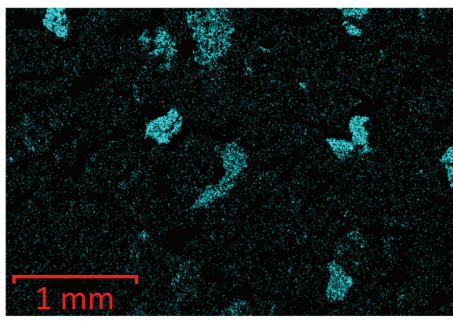

(e)

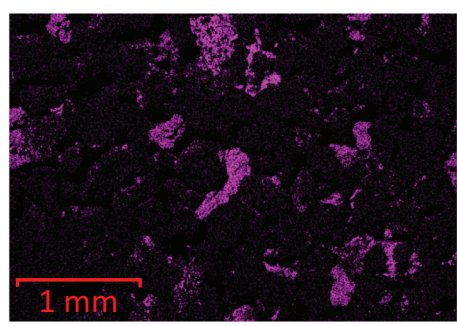

(c)

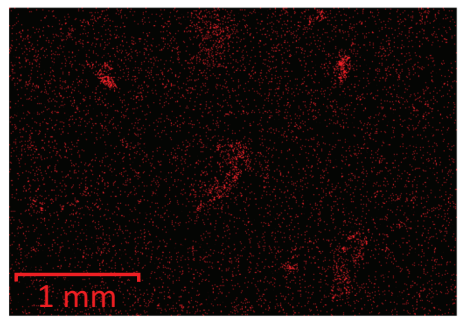

(f)

$\mathrm{Mg}$

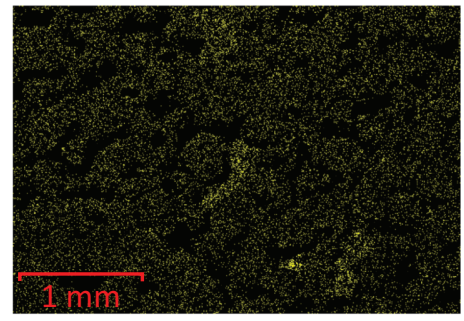

Figure 2 (a) Scanning electron microscopy (SEM) image of the polished surface of BS-V6. Energy dispersion spectrometry (EDS-SEM) images of the same sample portion showing the distribution of (b) potassium (K), (c) iron (Fe), (d) silicon (Si), (e) aluminium (Al) and (f) magnesium $(\mathrm{Mg})$. The data presented in this article are from experiments performed on sample BS-V5 cored from the same block as BS-V6. 
description of the experimental set-up and data processing. Pimienta et al. (2015a) and Pimienta, Fortin and Guéguen (2015b) provide further details on the calibration of the apparatus with standard materials. We will briefly list the governing relations used to determine the various moduli and associated attenuation modes.

Two types of stress oscillation on the sample, producing strains on the order of $\sim 10^{-6}$, can be performed in this cell: hydrostatic and axial. The hydrostatic oscillation $\left(4 \times 10^{-3}\right.$ to $\sim 1 \mathrm{~Hz}$ ) is induced by the confining pressure pump (Adelinet et al. 2010; David et al. 2013) and allows for directly measuring the sample's dynamic bulk modulus from the confining pressure oscillation $\Delta P_{c}=-\sigma_{i i} / 3$, where $\sigma_{i i}(i=1,2,3)$ are the principal stresses, and the associated volumetric strain $\varepsilon_{v o l}$ as follows:

$K_{\text {bydro }}=\frac{\Delta P_{c}}{\varepsilon_{v o l}}$.

The volumetric strain is determined from the average strain $\varepsilon_{\text {avg }}$ measured by eight strain gauges, comprising four pairs of radial and axial strain gauges, as $\varepsilon_{v o l}=3 \varepsilon_{\text {avg }}$.

The axial oscillation $\left(1 \times 10^{-1}\right.$ to $\left.\sim 30 \mathrm{~Hz}\right)$ is induced by a piezoelectric actuator placed between the sample and the axial piston of the cell and allows for measuring the sample's Poisson ratio $v$ and Young's modulus E:

$v=-\frac{\varepsilon_{r a d}}{\varepsilon_{a x}} \quad$ and $\quad E=\frac{\sigma_{a x}}{\varepsilon_{a x}}$,

where the axial stress $\sigma_{a x}$, is determined from the deformation of the aluminium end plate of known Young's modulus, and $\varepsilon_{\text {rad }}$ and $\varepsilon_{a x}$ are the average radial and axial strains on the sample. Given the Poisson ratio and Young's modulus, the axial bulk $K_{a x}$ and shear $G_{a x}$ moduli can be inferred as follows:

$K_{a x}=\frac{E}{3(1-2 v)} \quad$ and $\quad G_{a x}=\frac{E}{2(1+v)}$.

For each mode of deformation, the attenuation can be determined from the phase shift between the applied stress and resulting strain $\left(\Delta \phi=\phi_{\text {stress }}-\phi_{\text {strain }}\right)$. The bulk attenuation for the hydrostatic oscillation can be determined from the phase shift between the hydrostatic stress $\Delta P_{c}$ and the volumetric strain $\varepsilon_{\text {vol }}$, such that

$\Delta \phi_{\text {hydro }}=\phi_{\Delta P_{c}}-\phi_{\varepsilon_{v o l}}$.

The extensional mode attenuation is in turn determined from the phase shift between the axial stress $\sigma_{a x}$ and strain $\varepsilon_{a x}$, such that

$\Delta \phi_{\text {extensional }}=\phi_{\sigma_{a x}}-\phi_{\varepsilon_{a x}}$.
Assuming that the sample is isotropic, the bulk and shear attenuation can be inferred from the phase shift between the axial stress $\sigma_{a x}$ and the axial and radial strains $\varepsilon_{a x}$ and $\varepsilon_{\text {rad }}$ (Borgomano et al. 2017):

$\Delta \phi_{b u l k}=\phi_{\sigma_{a x}}-\phi_{\varepsilon_{a x}+2 \varepsilon_{r a d}} \quad$ and $\quad \Delta \phi_{\text {shear }}=\phi_{\sigma_{a x}}-\phi_{\varepsilon_{a x}-\varepsilon_{r a d}}$,

where the phases of $\left(\varepsilon_{a x}+2 \varepsilon_{\text {rad }}\right)$ and $\left(\varepsilon_{a x}-\varepsilon_{\text {rad }}\right)$ are derived from combining equations (2) and (3). The attenuation corresponding to each deformation mode can be calculated as (O’Connell and Budiansky 1978):

$Q^{-1}=\tan (\Delta \phi)$

\subsection{Experimental conditions}

Axial and hydrostatic oscillations, as well as ultrasonic wave velocity measurements, were performed on the dry and glycerine-saturated sample BS-V5. Axial oscillations were performed at effective stresses between 2.5 and $25 \mathrm{MPa}$, with an additional static axial load of $2 \mathrm{MPa}$. We will refer to the effective stress as the difference between the confining and fluid pressure, $\sigma_{e f f}=P_{c}-P_{f}$. The static axial load is applied to ensure coupling between the sample and the piezoelectric actuator. The hydrostatic oscillations were performed for the same range of effective stresses, however without imposing an additional static axial load. Before saturating with glycerine, a vacuum pump was used to remove air from the sample. Glycerine was then pumped into the sample using two Quizix pumps (Quizix Precision Pumps, Chandler Engineering, Tulsa, Oklahoma, USA) that subsequently regulated the fluid pressure at $4 \mathrm{MPa}$.

\section{RESULTS AND DISCUSSION}

\subsection{Measurement uncertainty}

To assess the uncertainty in our measured frequencydependent moduli and attenuation we look at the repeatability of the measurements and the variation of the data with respect to an idealized model. The moduli and attenuation are inferred from the strain measured on the sample. For hydrostatic forced oscillations we have eight measurements of strain, while for axial forced oscillations we have a pair of averages consisting of two axial and radial measurements of strain on opposing sides of the sample. We determine the repeatability of our moduli and attenuation for hydrostatic 

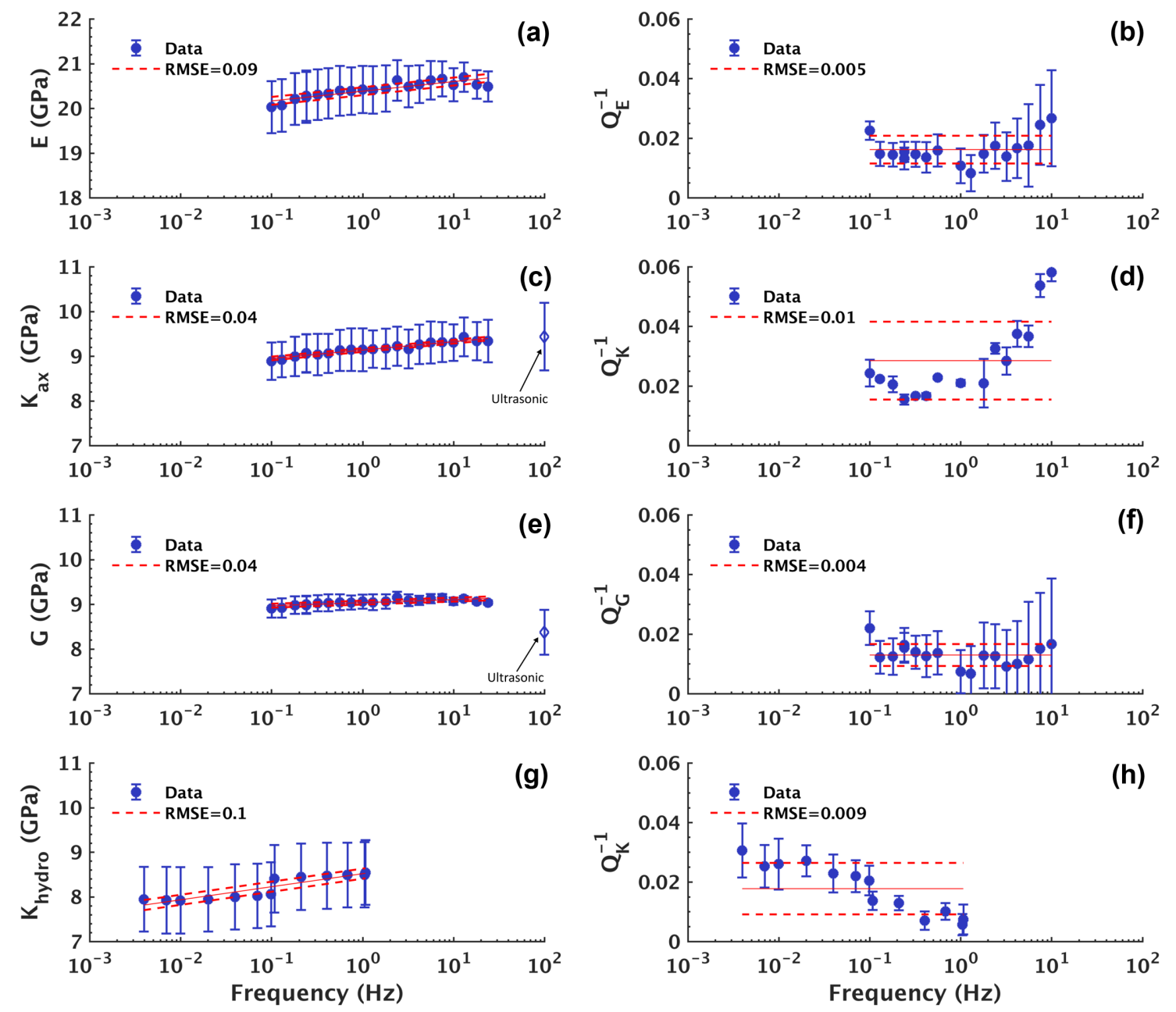

Figure 3 Frequency-dependent moduli and corresponding attenuation inferred from axial and hydrostatic forced oscillations on the dry sample at $5 \mathrm{MPa}$ effective stress. The data points represent the mean measurement and the error bars indicate the range of the measurements for axial oscillations $(\mathrm{a}-\mathrm{f})$ and the standard deviation in the measurements for hydrostatic oscillations $(\mathrm{g}-\mathrm{h})$. A linear function of $\log _{10}(\mathrm{frequency})$ is fit to the data and the root mean squared error (RMSE) is indicated in the legend. The solid line denotes the linear fit and the dashed line denotes the RMSE. The bulk and shear moduli inferred from the ultrasonic (1 MHz) P-and S-wave velocities are indicated by the diamond symbols (c and e).

oscillations by taking the standard deviation over the eight measurements of strain. In the case of the axial oscillations, we only have two measurements of each modulus and its corresponding attenuation, we therefore estimate the repeatability of our measurements from their range. To assess the variation of our data with respect to an idealized model we follow Adam, Batzle and Brevik (2006) procedure of fitting a linear function of $\log _{10}$ (frequency) to the mean of our measured data and determining the root mean squared error. A least-squares regression is used to fit the linear function to the data.

Figure 3 shows the moduli and corresponding attenuation inferred from axial and hydrostatic oscillations performed on the dry sample at $5 \mathrm{MPa}$ effective stress. The moduli increase moderately towards higher frequencies and the measurement range generally exceeds the variation of the measurement mean around the fit. The bulk and shear moduli are in agreement with the bulk and shear moduli inferred from the ultrasonic $(1 \mathrm{MHz}) \mathrm{P}$ - and $\mathrm{S}$-wave velocities and sample density. The uncertainty in the bulk and shear moduli inferred from the ultrasonic measurements is $\sim 8 \%$ and $\sim 6 \%$, respectively. For the corresponding attenuation the measurement range is much more variable and, for Young's and shear components, it increases towards higher frequencies. Attenuation is only shown up to $10 \mathrm{~Hz}$ because at higher frequencies it strongly deviates from the fitting 

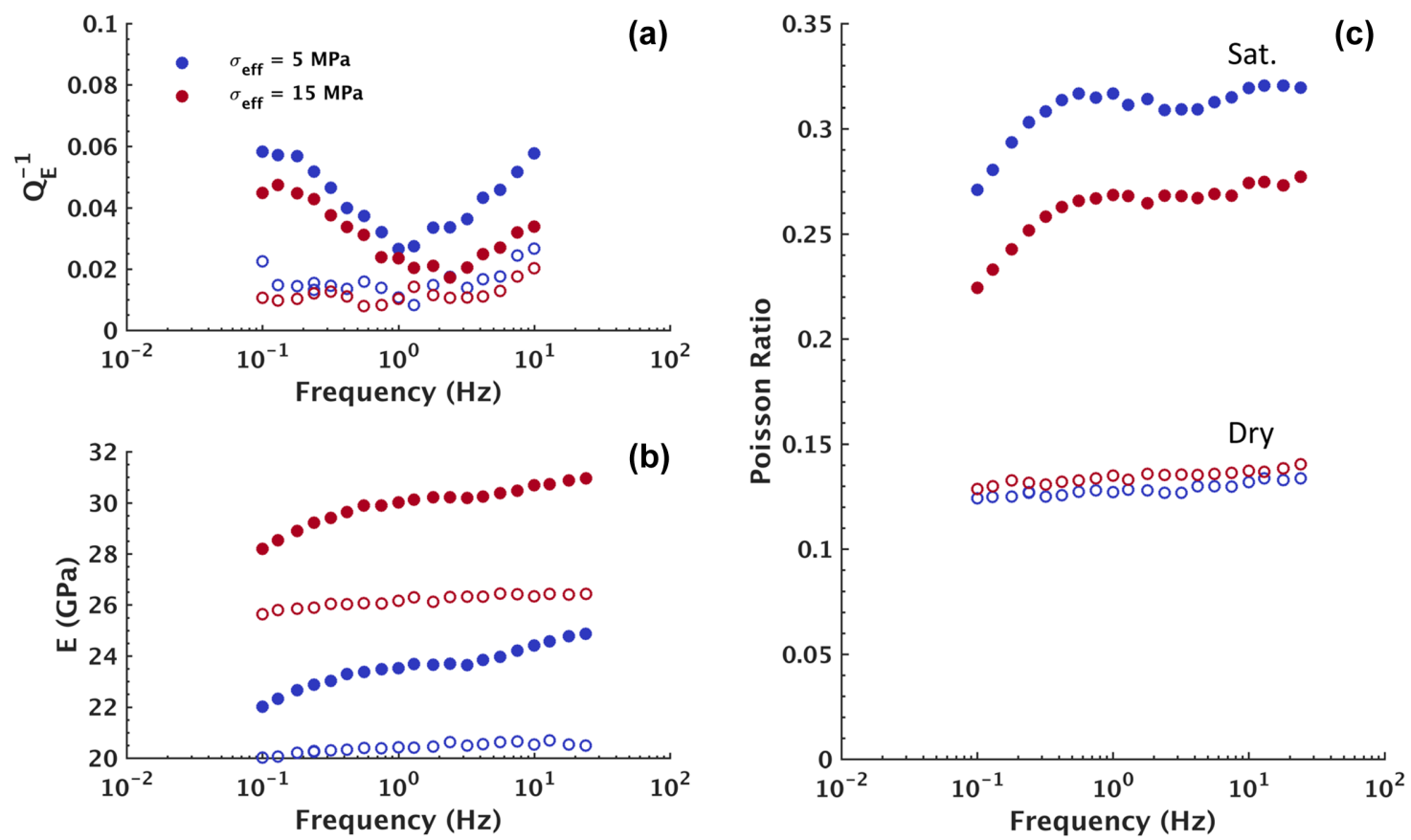

Figure 4 (a) Extensional attenuation $Q_{E}^{-1}$, (b) Young's modulus $E$ and (c) Poisson ratio for the dry and glycerine-saturated sample BS-V5 inferred from forced axial oscillations. The sample was subjected to a static axial stress of $2 \mathrm{MPa}$. The legend provides the applied effective stress $\sigma_{\text {eff }}$. Open symbols indicate the dry sample and filled symbols indicate the glycerine-saturated sample.

trend (Fig. 3b,d,f). In the following sections, we show the measurement range when error bars are displayed.

\subsection{Frequency dependence - glycerine saturation}

When the sample is glycerine saturated, all deformation modes show pronounced frequency-dependent attenuation (Figs. 4a, 5a and 6a). For the extensional (Fig. 4a) and bulk (Fig. 5a) attenuation, two peaks are observed, one at $\sim 0.1 \mathrm{~Hz}$ and another beginning at $\sim 3 \mathrm{~Hz}$ and above. For the shear attenuation (Fig. 6a), however, only the attenuation peak at higher frequencies is observed. The attenuation peak at $\sim 0.1 \mathrm{~Hz}$ is reduced in amplitude as the effective stress is increased. The second partial peak at higher frequencies is likewise reduced in amplitude, and at $15 \mathrm{MPa}$ effective stress the measured attenuation is comparable in amplitude to the attenuation measured in the dry sample.

As with the attenuation, the various stiffness moduli are frequency-dependent once the sample is saturated with glycerine (Figs. 4b, 5b and 6b). The overall increase in the sample's stiffness from dry-to-glycerine saturated is particularly observed in Young's modulus (Fig. 4b). The shear modulus at low frequencies is on the order of the shear modulus of the dry sample (Fig. 6b). Towards higher frequencies, the shear modulus shows some dispersion. Young's and bulk moduli are dispersive at both $\sim 0.1 \mathrm{~Hz}$ and again beginning at $\sim 3 \mathrm{~Hz}$. Overall the moduli become less dispersive with increasing effective stress. At high frequencies the bulk modulus possibly converges to a common limit, which is supported by the bulk modulus inferred from the ultrasonic P- and S-wave velocities. The Poisson ratio (Fig. 4c) is significantly increased with respect to the Poisson ratio measured in the dry sample and is frequency-dependent (Fig. 4c). For the saturated sample, the Poisson ratio is reduced with increasing effective stress and at high frequencies it is nearly frequency-independent.

Because the stress applied to the sample for the hydrostatic oscillation is determined from a pressure transducer in the confining oil, while for the axial oscillation the stress is determined from the deformation of the aluminium end plate on which the sample is placed, the bulk modulus and attenuation can be measured independently by these two methods. If the sample BS-V5 is in fact isotropic then the bulk modulus and attenuation measured by these two methods should be the same. In Fig. 5, we show the bulk modulus and attenuation determined from both hydrostatic and axial oscillations. We observe that the bulk modulus and attenuation are generally independent of the measurement type. However, as the effective stress is increased, the hydrostatic measurements 

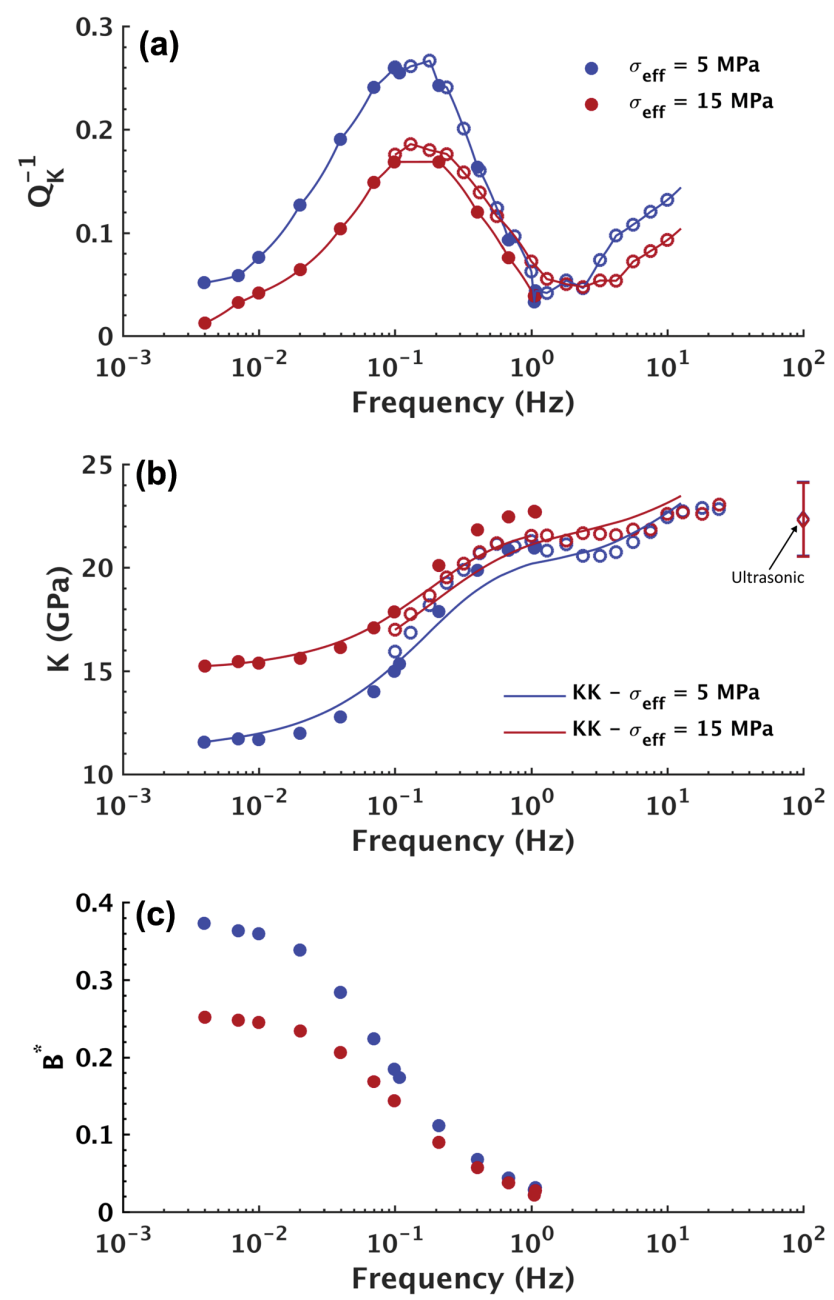

Figure 5 (a) Attenuation $Q_{K}{ }^{-1}$, (b) bulk modulus $K$ and (c) the pseudo-Skempton coefficient $B^{*}$ for the glycerine-saturated sample BS-V5 determined from forced axial (open symbols) and hydrostatic oscillations (filled symbols). The bulk modulus inferred from the ultrasonic $(1 \mathrm{MHz}) \mathrm{P}$ - and $\mathrm{S}$-wave velocities are indicated by the diamond symbols. Also shown are the results of Kramers-Kronig (KK) relations determined from a linear fit to the measured attenuation. For the axial oscillations, the sample was subjected to a static axial stress of $2 \mathrm{MPa}$. The legend provides the applied effective stress $\sigma_{\text {eff }}$.

do show a slightly higher bulk modulus than the axial measurements.

In the dry sample, our measurement uncertainty increased with frequency and we observed significant frequencydependent attenuation above $10 \mathrm{~Hz}$, indicating misalignments in the experimental set up or an inability of the piezoelectric actuator to generate a sinusoidal signal. To verify the quality of our measurements on the glycerine-saturated sample, we therefore use the Kramers-Kronig relations to check for the causality between our measured attenuation

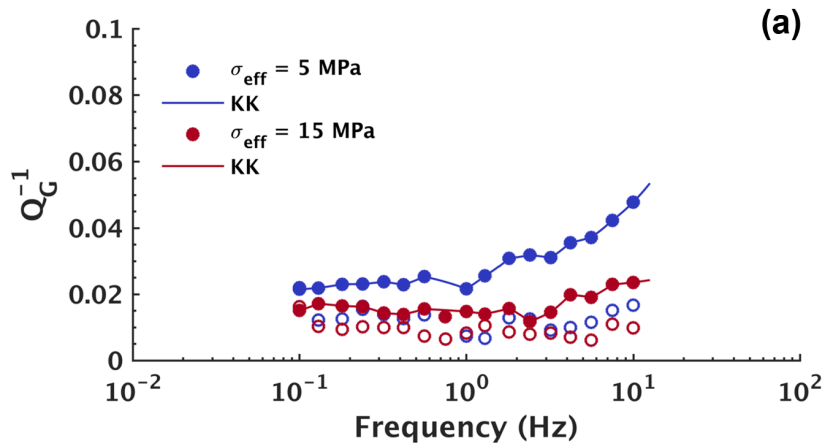

(b)

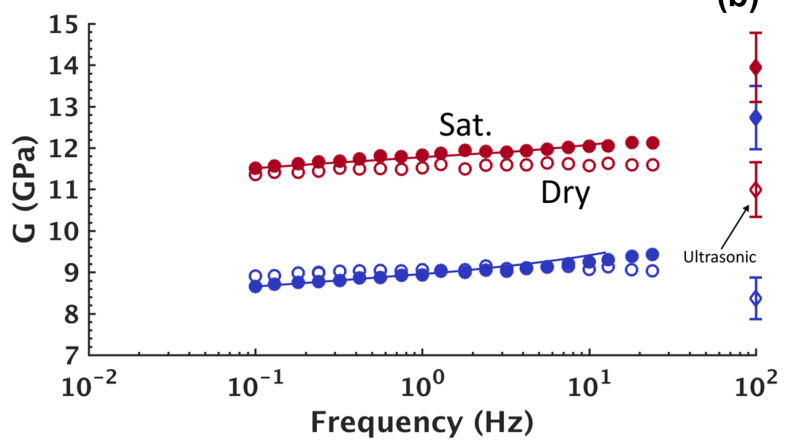

Figure 6 (a) Attenuation $Q_{G}{ }^{-1}$ and (b) shear modulus $G$ for the dry and glycerine-saturated sample BS-V5 determined from forced axial oscillations. The shear modulus inferred from the ultrasonic $(1 \mathrm{MHz})$ $\mathrm{S}$-wave velocities is indicated by the diamond symbols. Also shown are the results of Kramers-Kronig (KK) relations. The sample was subjected to a static axial stress of $2 \mathrm{MPa}$. The legend provides the applied effective stress $\sigma_{\text {eff }}$. Open symbols indicate the dry sample and filled symbols indicate the glycerine-saturated sample.

and moduli. The attenuation is fit with a linear interpolation to which we apply the Kramers-Kronig relations given by Mikhaltsevitch, Lebedev and Gurevich (2016). For the bulk (Fig. 5) and shear (Fig. 6) components we observe a satisfactory fit between the moduli and the respective attenuation.

The attenuation peak observed at $\sim 0.1 \mathrm{~Hz}$ is caused by the drained-undrained transition, which is a boundary condition problem of fluid-saturated samples associated with the measurement set-up (Dunn 1957; Gardner 1962; Moerig and Burkhardt 1989). The forced oscillation of the sample induces fluid pressure diffusion from the sample into the connecting pore fluid lines. The diffusion of pore fluid pressure can be described in terms of a pseudo-Skempton coefficient, defined as (Pimienta et al. 2015b):

$B^{*}=\frac{\Delta p_{f}}{\Delta p_{c}}$,

where $\Delta p_{f}$ is the fluid pressure amplitude measured in the pore fluid line and $\Delta P_{c}$ is the confining pressure amplitude. 
In Fig. 5(c) we see that the pseudo-Skempton coefficient is elevated at low frequencies, indicating that the glycerine had enough time to flow in response to the confining pressure oscillation and raise the pressure in the pore fluid lines. At low frequencies $(0.01 \mathrm{~Hz})$, the sample can therefore be considered partially drained. The pseudo-Skempton coefficient approaches zero as the frequency of the confining pressure oscillation increases because the fluid no longer has the time to diffuse form the sample and raise the pressure in the pore fluid lines. At high frequencies $(1 \mathrm{~Hz})$, the sample is therefore undrained. Increasing the effective stress increases the sample stiffness, which means that a larger portion of the load is carried by the frame of the sample and is not transferred to the fluid. The consequence of increasing the effective stress is that the pseudo-Skempton coefficient is reduced, which is consistent with the observations of Hart and Wang (1999) for the variation of the Skempton's coefficient with effective stress for Berea sandstone.

The second partial attenuation peak observed at above $\sim 3 \mathrm{~Hz}$ (Fig. 5a), on the other hand, is likely in response to squirt flow arising from microscopic compressibility heterogeneities in the rock. This is indicated in part by the sensitivity of the measured attenuation to an increase in effective stress resulting in a reduction of the compliant porosity and a consequent reduction in attenuation. Indicative of squirt flow is also the dispersion in the shear modulus and corresponding frequency-dependent attenuation (Fig. 6), which are not expected nor observed for the case of the drained-undrained transition.

\subsection{Theoretical models for drained-undrained transition and squirt flow}

A number of analytical solutions have been developed to explain modulus dispersion and attenuation related to squirt flow (e.g. Mavko and Jizba 1991; Chapman et al. 2002). Here we use Gurevich et al.'s (2010) analytical solution which describes the pressure diffusion between compliant and stiff pores for an oscillating stress. At low frequencies the solution converges to Gassmann's (1951) undrained limit, and at high frequencies it converges to Mavko and Jizba (1991) unrelaxed limit. The analytical solution assumes that the rock is isotropic, making it applicable to sample BS-V5, with pennyshaped compliant pores of a single aspect ratio. To investigate the drained-undrained transition, we in turn use a 1D analytical solution from Pimienta et al. (2016) for the FPD along the vertical axis of a sample subjected to a hydrostatic pres-
Table 2 Rock and fluid properties for the simple isotropic squirt-flow model for sample BS-V5 subjected to an effective stress of $5 \mathrm{MPa}$

\begin{tabular}{llll}
\hline & Parameters & Symbol & Model input \\
\hline \multirow{2}{*}{ Fluid } & Fluid bulk modulus & $K_{f}$ & $4.36 \mathrm{GPa}$ \\
& Viscosity & $\eta$ & $1.087 \mathrm{~Pa} \cdot \mathrm{s}$ \\
\multirow{4}{*}{ Rock } & Stiff porosity & $\phi_{s}$ & 0.2213 \\
& Compliant porosity & $\phi_{c}$ & $1.55 \times 10^{-4}$ \\
& Grain bulk modulus & $K_{g}$ & $36 \mathrm{GPa}$ \\
& Drained bulk modulus & $K_{d}$ & $9.2 \mathrm{GPa}$ \\
& Drained shear modulus & $G_{d}$ & $9.1 \mathrm{GPa}$ \\
& High-pressure bulk modulus & $K_{b}$ & $13.3 \mathrm{GPa}$ \\
& Crack aspect ratio & $\alpha$ & 0.0025 \\
\hline
\end{tabular}

sure oscillation. At high frequencies, the solution converges to Gassmann's (1951) undrained limit.

The input parameters for both models are given in Tables 2 and 3, and correspond to a sample subjected to an effective stress of $5 \mathrm{MPa}$. The drained bulk and shear moduli are measured from the forced axial oscillations on the dry sample. The compliant porosity, $\phi_{c}$, was estimated from the volumetric strain measured on the dry sample, following the procedure described in Appendix A of Gurevitch et al. (2010). The high-pressure bulk modulus required by the squirt-flow model corresponds to the bulk modulus measured at $25 \mathrm{MPa}$ confining pressure from the forced axial oscillations on the dry sample. The aspect ratio of the compliant pores cannot be accurately determined from the mechanical and scanning electron microscopy (SEM) data and is therefore used as a fitting parameter, as it mainly impacts the characteristic frequency where the maximum attenuation occurs. A first-order estimate of the aspect ratio can be obtained from Walsh (1965):

$\alpha \approx \frac{4 P_{\text {closure }}\left(1-v^{2}\right)}{\pi E}$,

Table 3 Rock and fluid properties for the 1D model of the drainedundrained transition for sample BS-V5 subjected to an effective stress of $5 \mathrm{MPa}$

\begin{tabular}{llll}
\hline & Parameter & Symbol & Model input \\
\hline \multirow{2}{*}{ Fluid } & Fluid bulk modulus & $K_{f}$ & $4.36 \mathrm{GPa}$ \\
\multirow{3}{*}{ Rock } & Viscosity & $\eta$ & $1.087 \mathrm{~Pa} \cdot \mathrm{s}$ \\
& Length & $L$ & $0.083 \mathrm{~m}$ \\
& Diameter & $D$ & $0.04 \mathrm{~m}$ \\
& Porosity & $\phi$ & 0.2215 \\
& Permeability & $\kappa$ & $58.7 \mathrm{mD}$ \\
\multirow{2}{*}{ Dead volume } & Grain bulk modulus & $K_{g}$ & $36 \mathrm{GPa}$ \\
& Drained bulk modulus & $K_{d}$ & $9.2 \mathrm{GPa}$ \\
& Dead volume & $V_{\text {dead vol. }}$ & $26 \times 10^{-6} \mathrm{~m}^{3}$ \\
\hline
\end{tabular}


where $P_{\text {closure }}$ is the confining pressure at which the compliant cracks closed in the dry sample, while $E$ is Young's modulus and $v$ is Poisson's ratio. Considering a closure pressure of around $25 \mathrm{MPa}$, at which we measured a Young's modulus of $\sim 28 \mathrm{GPa}$ and Poisson's ratio of $\sim 0.14$, we can infer that the characteristic aspect ratio should be on the order of $\sim 1 \times$ $10^{-3}$, which is consistent with our choice of aspect ratio (Table 2).

Figure 7 shows the combined result of the two analytical models together with the moduli and attenuation measured on the dry and glycerine-saturated sample at $5 \mathrm{MPa}$ effective stress. The results of both models are simply added to reach the combined result, however it should be noted that this assumes that the two mechanisms are independent of each other. Furthermore, neither model accounts for the intrinsic attenuation observed in the dry sample. Then, also assuming it to be independent of the other two attenuation mechanisms, we add the mean attenuation measured in the dry sample to the model result (Tisato and Quintal 2013, 2014). Young's modulus (Fig. 7a) and attenuation (Fig. 7b) derived from the models reproduce well the laboratory observation, while the bulk modulus dispersion (Fig. 7c) and attenuation (Fig. 7d) are slightly underestimated. The shear modulus measured in the glycerine-saturated sample is reduced in relation to the dry sample (Fig. 7e) and the model therefore does not fit it as well. On the other hand, the shear attenuation (Fig. $7 \mathrm{f}$ ) is reasonably well reproduced. However, with respect to the bulk and shear moduli inferred from the ultrasonic Pand $\mathrm{S}$-wave velocities, the squirt-flow model underestimates the unrelaxed limit (Figs. 5 and 6). The shear modulus inferred from the ultrasonic velocities is $12.7 \mathrm{GPa}$, while the squirt-flow model predicts an unrelaxed limit of 9.9 GPa.

\subsection{Comparison with previous experimental results}

Our experiments are very similar to those performed by Mikhaltsevitch, Lebedev and Gurevich (2015) and Mikhaltsevitch et al. (2016), also performed on a Berea sandstone sample fully saturated with glycerine. Their sample D had a permeability of $71 \mathrm{mD}$, a porosity of $19 \%$ and while composed mainly of quartz $(80 \%)$ and feldspar $(12 \%)$ also had substantial amounts of kaolinite $(8 \%)$, making it similar to our sample BS-V5. In their study, the forced axial oscillation measurements were performed on both the dry and glycerine-saturated sample at $10 \mathrm{MPa}$ effective stress. Under glycerine saturation, the pore pressure was maintained at 3 $\mathrm{MPa}$. The measurements were performed at different temperatures ranging from $23^{\circ} \mathrm{C}$ to $31^{\circ} \mathrm{C}$. For the sake of comparing our data to theirs, we will only consider the measurements performed at $23^{\circ} \mathrm{C}$. Mikhaltsevitch et al. (2015) are confident that their measurements are performed under undrained conditions, based on Gassmann's predictions, matching well their measured bulk modulus at low frequencies. They interpret the observed frequency-dependent attenuation to be in response to squirt flow.

In Fig. 8, we compare Young's modulus and attenuation measured in the dry and glycerine-saturate sample D of Mikhaltsevitch et al. (2016) (Fig. 8a,b) to that measured in our sample BS-V5 (Fig. 8c,d). It is important to note that we are showing our measurements performed at $5 \mathrm{MPa}$ effective stress because we did not perform measurements at $10 \mathrm{MPa}$ effective stress. However, as seen in Section 3.2, we observed that increasing the effective stress reduces the attenuation amplitude, but does not result in a large shift of the attenuation peaks with respect to frequency. While sample $\mathrm{D}$ is more compressible, under dry conditions it is less attenuating than sample BS-V5, which could indicate for our sample a minor dependence on the strain amplitude (e.g. Gordon and Davis 1968; Winkler, Nur and Gladwin 1979; Tisato and Quintal 2014). However frequency-dependent attenuation in response to FPD should be largely independent of strain. Mikhaltsevitch et al. (2016) observe the attenuation peak that they attribute to squirt flow at $\sim 0.4 \mathrm{~Hz}$ (Fig. $8 \mathrm{~b}$ ), while for our sample the attenuation peak that we attribute to squirt flow is at $>10 \mathrm{~Hz}$ (Fig. 8d). The separation of these two attenuation peaks by $1-$ 2 orders of magnitude can be explained by a relatively minor variation in the characteristic aspect ratio of the compliant pores in the sample, given that the characteristic frequency of squirt flow is proportional to the cube of the aspect ratio (O’Connell and Budiansky 1977; Gurevich et al. 2010).

Included in Fig. 8(a,b), we show the result of Gurevich et al.'s (2010) squirt-flow model and in Fig. 8(c,d) we show the combined result of the squirt-flow model and Pimienta et al.'s (2016) model for the drained-undrained transition. As input parameters, we use the bulk and shear modulus and the porosity of the dry sample D (Mikhaltsevitch et al. 2015). The other rock parameters for the model were not available, therefore we use those used for sample BS-V5 (Table 2). The aspect ratio of compliant pores is again used as a fitting parameter, with the value of 0.0005 which is lower than the one chosen for BS-V5, for which an aspect ratio of 0.0025 was chosen. Both Young's modulus (Fig. 8a) and attenuation (Fig. $8 \mathrm{~b}$ ) are underestimated by the analytical solution, which is possibly related to the choice of the high-pressure bulk modulus that controls the bulk modulus dispersion. The analytical solution does not account for a distribution of aspect ratios 

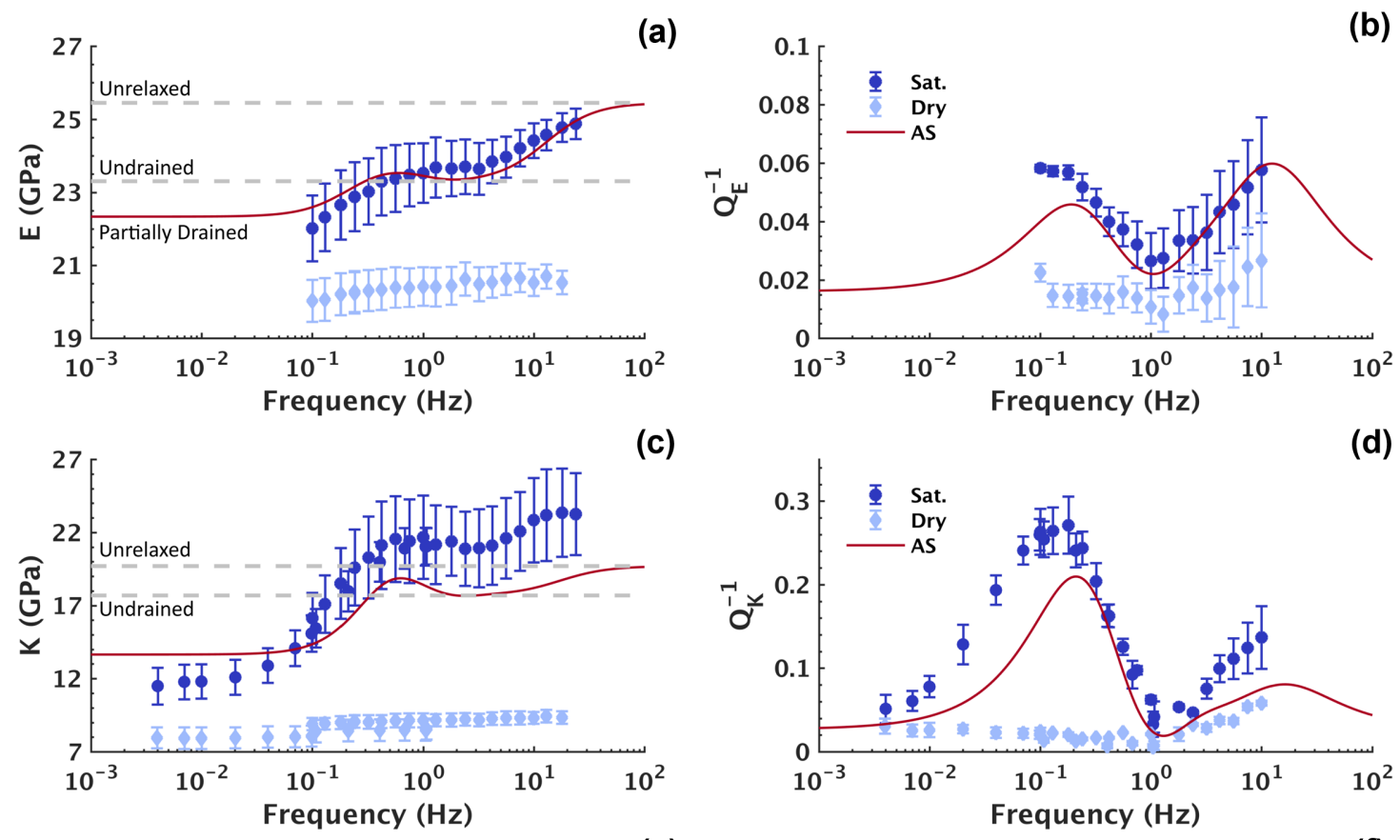

(e)
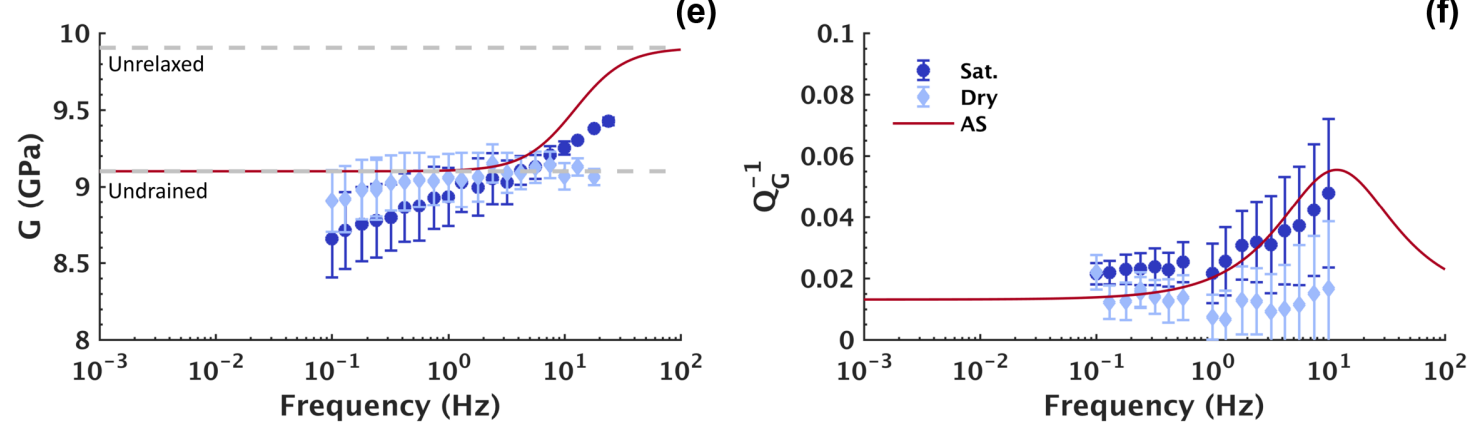

Figure 7 Moduli and attenuation for the dry and glycerine-saturated sample BS-V5 at $5 \mathrm{MPa}$ effective stress determined from both axial and hydrostatic oscillations (already shown in Figs. 5,6) together with the combined results of the analytical solutions (AS) for the drained-undrained transition and squirt flow.

and therefore the asymptote of attenuation at low-frequency scales as $Q^{-1} \propto f$, while at high-frequency scales as $Q^{-1} \propto$ $f^{-1}$ (Gurevich et al. 2010). The measured attenuation shows a significantly broader curve than the result of the analytical solution suggesting that sample $\mathrm{D}$ has a distribution of aspect ratios as would be expected for sandstone (e.g. Cheng and Toksöz 1979; Subramaniyan et al. 2015). In sample BS-V5 (Fig. 8c,d), it is not clear whether the sample has a narrower distribution in aspect ratios, given that the attenuation curve is only partially observed.

Berea sandstone has been extensively studied in the past and it contains substantial amounts of clay, which fills pores and coats grains (e.g. Kareem et al. 2017). Christensen and Wang (1985) observed an increase in compressional wave velocities and a decrease in shear wave velocities with pore pressure in water-saturated Berea sandstone, attributing the observations to the high compressibility of clays that make up parts of the cement. Zoback and Byerlee (1975) also attribute the compressibility of clay to the increase in permeability with increasing pore pressure in Berea sandstone. At seismic frequencies Pimienta et al. (2017) observe a sensitivity of the frequency-dependent Poisson ratio to fluid pressure in a Berea sandstone sample saturated with a glycerine-water mixture when varying the fluid pressures to up to $9 \mathrm{MPa}$ at a constant effective stress of $1 \mathrm{MPa}$. However, they do not elaborate on what may be inducing the increase in Poisson's ratio with fluid pressure. Although the experiments carried out in our study are very similar to those of Mikhaltsevitch et al. (2016) in 

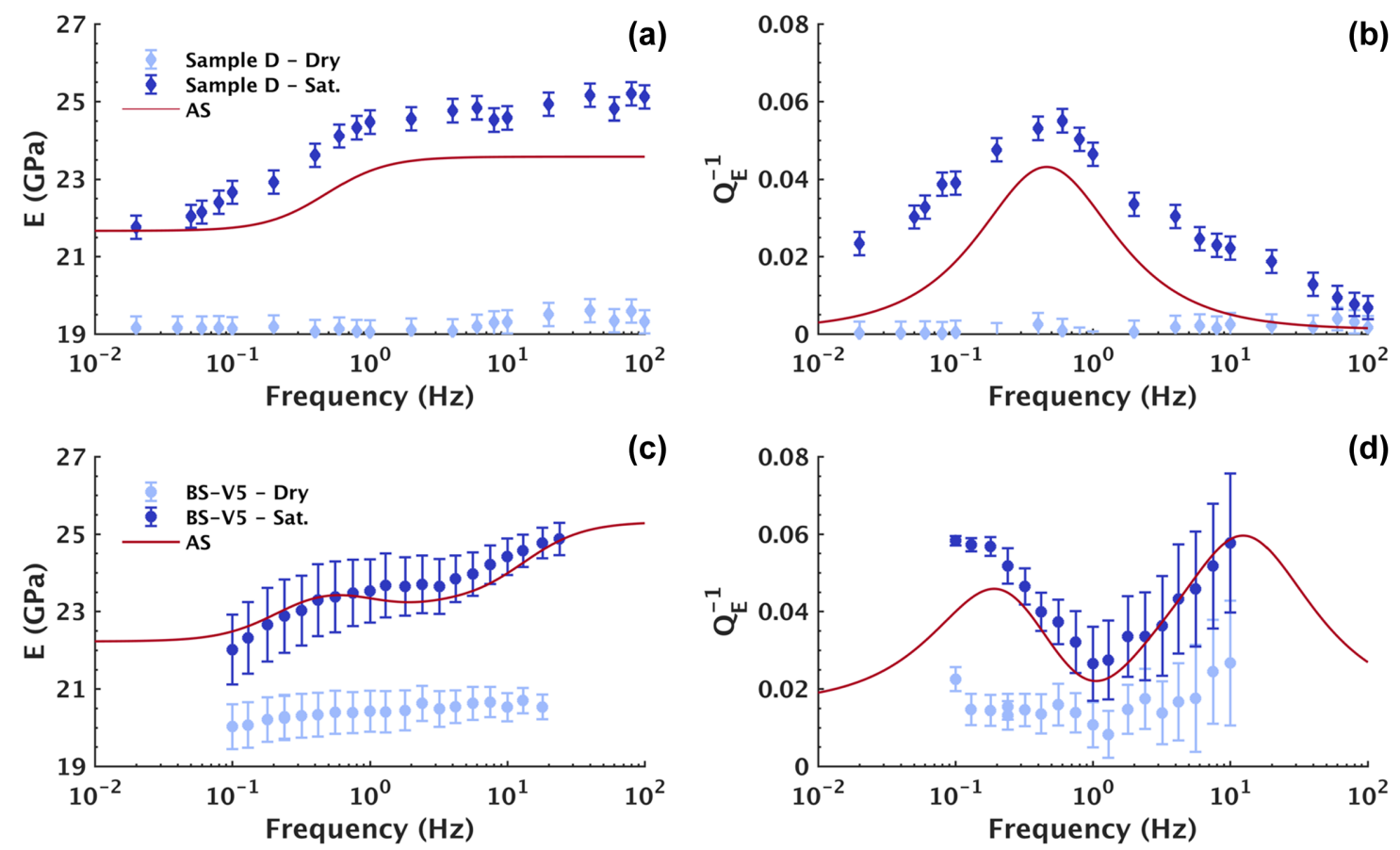

Figure 8 (a) Young's modulus and (b) attenuation $Q_{E}^{-1}$ for the dry and glycerine-saturated Berea sandstone (sample D) measured at $10 \mathrm{MPa}$ effective stress by Mikhaltsevitch et al. (2016), together with the results of the analytical solution (AS) for squirt flow. For comparison, our measurements for c) Young's modulus and d) attenuation $Q_{E}^{-1}$ of the dry and glycerine-saturated sample BS-V5 at 5 MPa effective stress (already shown in Fig. 4), together with the results of the analytical solutions for the drained-undrained transition and squirt flow.

terms of rock type and fluid used and the range of effective stresses applied, the fluid pressure was $4 \mathrm{MPa}$ in sample BSV5 and $3 \mathrm{MPa}$ in sample D. The difference in fluid pressure is not very large, however, for future experimental research on squirt flow, the impact of changes in fluid pressure could be interesting because of the sensitivity of this phenomenon to the aspect ratio of compliant pores. In Berea sandstone, where clays coat the grains, a variation in fluid pressure at constant effective stress could possibly facilitate a change in aspect ratio of compliant pores, which could in turn be identified in the measured frequency dependence of attenuation and modulus dispersion.

\section{CONCLUSIONS}

We performed hydrostatic and axial forced oscillation experiments on a dry and glycerine-saturated Berea sandstone sample. In the glycerine-saturated sample, the measured attenuation is frequency-dependent with an attenuation peak at $\sim 0.1 \mathrm{~Hz}$ and a second, partial, peak beginning at $\sim 3 \mathrm{~Hz}$. The first attenuation peak is in response to fluid pressure diffusion (FPD) from the sample into the pore fluid lines, referred to as the drained-undrained transition. The second partial attenuation peak is likely in response to squirt flow, resulting from fluid pressure diffusion associated with microscopic heterogeneities in the compressibility of the solid frame of the rock. The results of analytical solutions for the drained-undrained transition and squirt flow reproduce fairly well the corresponding measured attenuation and moduli dispersion. A comparison with independently conducted experiments on a similar Berea sandstone sample under comparable conditions suggest the high sensitivity of squirt flow to variations in the characteristic aspect ratios of the compliant porosity.

\section{ACKNOWLEDGEMENTS}

This work was supported by a grant from the Swiss National Science Foundation. We thank Marie Violay for access to the EPFL Laboratory of Experimental Rock Mechanics, Laurent Gastaldo for coring the samples from the source blocks and Lucas Pimienta for doing the porosity measurements. We thank Jean-Pierre Burg for access to the ETH Zurich Rock Deformation Laboratory and Claudio Madonna for helping with the sample preparation. The data for this article are available by contacting the authors. 


\section{ORCID}

Jan V. M. Borgomano

http://orcid.org/0000-0002-6332-1878

Hanjun Yin (iD http://orcid.org/0000-0001-7888-2240

Beatriz Quintal (D) http://orcid.org/0000-0001-6714-420X

\section{REFERENCES}

Adam L., Batzle M. and Brevik I. 2006. Gassmann fluid substitution and shear modulus variability in carbonates at laboratory seismic and ultrasonic frequencies. Geophysics 71, F173-F183.

Adelinet M., Fortin J. and Gueguen Y. 2011. Dispersion of elastic moduli in a porous-cracked rock: theoretical predictions for squirtflow. Tectonophysics 503, 173-181.

Adelinet M., Fortin J., Gueguen Y., Schubnel A. and Geoffroy L. 2010. Frequency and fluid effects on elastic properties of basalt: experimental investigations. Geophysical Research Letters 37, L02303.

Borgomano J.V.M., Pimienta L., Fortin J. and Gueguen Y. 2017. Dispersion and attenuation measurements of elastic moduli of a dual-porosity limestone. Journal of Geophysical Research: Solid Earth 122, 2690-2711.

Chapman M., Zatspin S.V. and Crampin S. 2002. Derivation of a microstructural poroelastic model. Geophysical Journal International 151, 427-451.

Chapman S. and Quintal B. 2018. Numerical analysis of local strain measurements in fluid saturated rocks submitted to forced oscillations. Geophysics 83, MR309-MR316.

Cheng C.H. and Toksöz M.N. 1979. Inversion of seismic velocities for the pore aspect ratio spectrum of rock. Journal of Geophysical Research 84, 7533-7543.

Christensen N.I. and Wang H.F. 1985. The influence of pore pressure and confining pressure on dynamic elastic properties of Berea sandstone. Geophysics 50, 207-213.

David E.C., Fortin J., Schubnel A., Gueguen Y. and Zimmerman R.W. 2013. Laboratory measurements of low- and high-frequency elastic moduli in Fontainebleau sandstone. Geophysics 78, D369-D379.

Dunn K.-J. 1987. Sample boundary effect in acoustic attenuation of fluid-saturated porous cylinders. Journal of the Acoustical Society of America 81, 1259-1266.

Gardner G.H.F. 1962. Extensional waves in fluid-saturated porous cylinders. Journal of the Acoustical Society of America 34, 36-40.

Gassmann F. 1951. Über die Elastizität poröser Medien: Vierteljahrsschrift der Naturforschenden Gesellschaft in Zürich, 96, 1-23.

Gordon R.B. and Davis L.A. 1968, Velocity and attenuation of seismic waves in imperfectly elastic rock. Journal of Geophysical Research 73, 3917-3935.

Gurevich B., Makaryska D., de Paula O.S. and Pervukhina M. 2010. A simple model for squirt-flow dispersion and attenuation in fluidsaturated granular rocks. Geophysics 75, 6 .

Hart D.J. and Wang H.F. 1999. Pore pressure and confining stress dependence of poroelastic linear compressibilities and Skempton's B coefficient for Berea sandstone. 37th US Rock Mechanics Symposium. American Rock Mechanics Association.
Jones T.D. 1986. Pore fluids and frequency-dependent wave propagation in rocks. Geophyics 51, 1939-1953.

Kareem R., Cubillas P., Gluyas J., Bowen L., Hillier S. and Greenwell H.C. 2017. Multi-technique approach to the petrophysical characterization of Berea sandstone core plugs (Cleveland Quarries, USA). Journal of Petroleum Science and Engineering 147, 436-455.

Masson Y.J. and Pride S.R. 2007. Poroelastic finite difference modeling of seismic attenuation and dispersion due to mesoscopic-scale heterogeneity. Journal of Geophysical Research 112, 1-17.

Masson Y.J. and Pride S.R. 2011. Seismic attenuation due to patchy saturation. Journal of Geophysical Research 116, 3206-3206.

Mavko G. and Jizba D. 1991. Estimating grain-scale fluid effects on velocity dispersion in rocks. Geophysics 56, 1940-1949.

McKavanagh B. and Stacey F.D. 1974. Mechanical hysteresis in rocks at low strain amplitudes and seismic frequencies. Physics of the Earth and Planetary Interiors 8, 246-250.

Mikhaltsevitch V., Lebedev M. and Gurevich B. 2015. A laboratory study of attenuation and dispersion effects in glycerol-saturated Berea sandstone at seismic frequencies. SEG Technical Program Expanded Abstractsv, 3085-3089.

Mikhaltsevitch V., Lebedev M. and Gurevich B. 2016. Validation of the laboratory measurements at seismic frequencies using the Kramers-Kronig relationship. Geophysical Research Letters 43, 4986-4991.

Mörig R. and Burkhardt H. 1989. Experimental evidence for the Biot-Gardner theory. Geophysics 54, 524-527.

Müller T.M., Toms-Stewart J. and Wenzlau F. 2008. Velocitysaturation relation for partially saturated rocks with fractal pore fluid distribution. Geophysical Research Letters 35, L09306.

O'Connell R.J. and Budiansky B. 1977. Viscoelastic properties of fluid-saturated cracked solids. Journal of Geophysical Research 82, 5719-5735.

O’Connell R.J. and Budiansky B. 1978. Measures of dissipation in viscoelastic media. Geophysical Research Letters 5, 5-8.

Pimienta L., Borgomano J.V.M., Fortin J. and Guéguen Y. 2016. Modelling the drained/undrained transition: effect of the measuring method and the boundary conditions. Geophysical Prospecting 64, 1098-1111.

Pimienta L., Borgomano J.V.M., Fortin J. and Guéguen Y. 2017. Elastic dispersion and attenuation in fully saturated sandstones: role of mineral content, porosity, and pressures. Journal of Geophysical Research: Solid Earth 122, 9950-9965.

Pimienta L., Fortin J. and Guéguen Y. 2015a. Experimental study of Young's modulus dispersion and attenuation in fully saturated sandstones. Geophysics 80, L57-L72.

Pimienta L., Fortin J. and Guéguen Y. 2015b. Bulk modulus dispersion and attenuation in sandstones. Geophysics 80, D111-D127.

Pride S.R., Berryman J.G. and Harris J.M. 2004. Seismic attenuation due to wave-induced flow. Journal of Geophysical Research 109, B01201.

Spencer, J.W. and Shine J. 2016. Seismic wave attenuation and modulus dispersion in sandstones. Geophysics 81, D211-D231.

Subramaniyan S., Quintal B., Madonna C. and Saenger E.H. 2015. Laboratory-based seismic attenuation in Fontainebleau sandstone: evidence of squirt flow. Journal of Geophysical Research: Solid Earth 120, 7526-7535. 
Tisato N. and Quintal B. 2013. Measurements of seismic attenuation and transient fluid pressure in partially saturated Berea sandstone: evidence of fluid flow on the mesoscopic scale. Geophysical Journal International 195, 342-351.

Tisato N. and Quintal B. 2014. Laboratory measurements of seismic attenuation in sandstone: strain versus fluid saturation effects, Geophysics 79, WB9-WB14.
Walsh J.B. 1965. The effects of cracks on the compressibility of rock. Journal of Geophysical Research 70, 381-389.

Winkler K., Nur A. and Gladwin M. 1979. Friction and seismic attenuation in rocks. Nature 277, 528-531.

Zoback M.D. and Byerlee J.D. 1975. Permeability and effective stress. Bulletin - American Association of Petroleum Geologists 59, 154158. 